\title{
Special section on software engineering and formal methods: extended versions of contributions presented at SEFM 2008
}

\author{
Antonio Cerone · Stefan Gruner
}

Published online: 23 July 2010

(C) Springer-Verlag 2010

\section{Introduction}

Originally motivated by the need to increase the reliability and robustness of a software and hardware design a few decades ago, formal methods have now established themselves as a distinct important area of computer science. However, the original software engineering motivation of formal methods has been largely weaken throughout the years by the very high cost of their application to system development. As a result, formal methods are now moving towards two different directions, involving two fundamentally distinct research communities. On one side are those who are interested in theoretical aspects of formal methods and focus on the definition of complex mathematical frameworks, which are only loosely inspired by practical problems. On the other side are those who are interested in looking for practical problems, not just in software and hardware development but also in the application areas such as system biology and chemistry, where using formal methods is expected to be worthy in terms of time, effort and financial cost.

This Special Section is dedicated to the memory of R. Geoff Dromey (1946-2009), who was one of the founders and a Steering Committee member of the SEFM conference series.

\footnotetext{
A. Cerone $(\varangle)$

International Institute for Software Technology,

United Nations University, Macau, SAR, China

e-mail: antonio@iist.unu.edu

S. Gruner

Department of Computer Science, Universiteit van Pretoria, Pretoria, Republic of South Africa

e-mail: sg@cs.up.ac.za
}

A larger fragmentation occurs within these two main formal methods communities because of the increasing complexity of the mathematical machineries used by the theoretical community and because of the very specialised application domains considered by the practical community. The final result is the fragmentation of the formal methods community into a miriad of small communities, who use their own jargon, often ununderstandable to outsiders, and have their own conferences and other closed events.

This progressive detachment from the original practical motivation as well as the fragmentation in small communities has prevented formal methods from having a large diffusion outside Europe and North America, with little or no dissemination at all in developing countries.

\section{The SEFM conference series}

The IEEE International Conferences on Software Engineering and Formal Methods address the global formal methods community encouraging researchers to emphasise practical applications to software engineering rather than purely theoretical aspects of formal methods. They aim to bring together practitioners and researchers from academia, industry and government to advance the state of the art in formal methods to scale up their application in software industry and to encourage their integration with practical engineering methods.

An important aim of the SEFM conferences is to encourage research cooperation between developing countries and industrialised countries all over the world. For this reason, SEFM conferences are alternatively held in countries where formal methods have a long-dated research tradition, such as Australia, Germany, UK and Italy, and countries, which usually belong to the developing world, such as China, India, 
South Africa and Vietnam, where little research in formal methods is carried out. The first SEFM conference was held in Brisbane, Australia, in 2003. Subsequent SEFM conferences were held in Beijing, China, in 2004, Koblenz, Germany, in 2005, Pune, India, in 2006, London, UK, in 2007, Cape Town, South Africa, in 2008 and Hanoi, Vietnam, in 2009. Next SEFM conference will be held in Pisa, Italy.

\section{Selection process}

This Special Section is devoted to extended versions of selected contributions that have been presented at the Sixth IEEE International Conference on Software Engineering and Formal Methods (SEFM 2008). Supported by the IEEE Computer Society, Formal Methods Europe (FME) and the International Institute for Software Technology of the United Nations University (UNU-IIST), SEFM 2008 had taken place on the premises of the University of Cape Town, South Africa, from 10 to 14 November 2008. The conference proceedings include 38 peer-reviewed papers ( 33 regular papers and 5 short papers), 2 invited keynote lectures and 1 tutorial [1]. The 38 peer-reviewed papers have been selected out of 115 submissions, with approximately $33 \%$ acceptance rate for SEFM 2008 [1].

After the conference, we invited the authors of the 12 best contributed papers presented at SEFM 2008 to submit extended versions of their papers. The submitted extended papers were subject to another rigorous process of reviewing and improvement with up to three iterations of revision. Finally, only 5 papers were chosen to represent the 'essence' of SEFM 2008 in this journal. With respect to the conference proceedings [1] the acceptance rate is thus approximately 13\%; with respect to the initial 115 submissions the acceptance rate is approximately $4 \%$.

\section{Summaries of the articles}

\subsection{Formalizing requirements with object models and temporal constraints}

Stefano Tonetta, Alessandro Cimatti, Marco Roveri and Angelo Susi introduce a formalism for the representation of object models that supports reasoning about their temporal evolution. This formalism is the basis for a novel approach to the formal representation and validation of requirements. The approach, which has been used in an industrial software project by the authors, allows to check whether requirements are consistent, are compatible with some scenarios, and guarantee some implicit properties.

\subsection{An algebra of product families}

Bernhard Möller, Peter Höfner and Ridha Khedri use the well established mathematical structure of idempotent semirings as the basis for a product family algebra that supports formal description and analysis of the commonalities and variabilities of a system family. An important application of this algebra concerns the multi view reconciliation problem which arises when complex systems are modelled. The authors use algebraic integration constraints linking features in one view to features in the same or a different view and demonstrate in several examples the suitability of this approach for a wide class of integration constraint formulations.

\subsection{Büchi automata for modeling component connectors}

Marcello Bonsangue, Mohammad Izadi and Dave Clarkepropose a novel operational semantics based on Büchi automata for Reo, a coordination language based on connectors for the orchestration of components in a component based systems. Such a semantics, being based on standard automata theory, promises to support the use of existing theories and tools in the analysis of the component based system.

\subsection{Testing timed systems modeled by stream x-machines}

Mercedes Merayo, Manuel Nunez and Robert Hierons define a timed extension for Stream X-machines, a formalism to specify real systems with complex data structures. They then propose a formal testing methodology, based on Timed Stream X-machines, to systematically test a system with respect to a specification, and present an algorithm to derive sound and complete test suites with respect to three conformance relations.

\subsection{Precise null-pointer analysis}

Fausto Spoto uses abstract interpretation to build and prove correct a first, flow and context-sensitive static null-pointer analysis for Java bytecode which infers non-null annotations. His approach is based on Boolean formulae, implemented with binary decision diagrams, deals with exceptions, which is not the case of most other approaches to null-pointer analysis, and is shown through experiments to be faster and more precise than another correct null-pointer analysis from the literature.

Acknowledgments We would like to thank all SEFM 2008 authors who have submitted their works to be reviewed for this special section. We are very grateful to all reviewers for their thorough and thoughtful assessments of the submitted papers and for their help throughout the selection process. Last but not least we would like to thank Bernhard Rumpe and Martin Schindler, from the editorial office of Software and System Modeling, for having made this special section possible. 


\section{Reference}

1. Cerone, A., Gruner, S. (eds.): Proceedings of the Sixth IEEE International Conference on Software Engineering and Formal Methods (SEFM 2008), Cape Town, South Africa, IEEE Computer Society Press, 10-14 November 2008. ISBN 978-0-7695-3437-4

\section{Author Biographies}

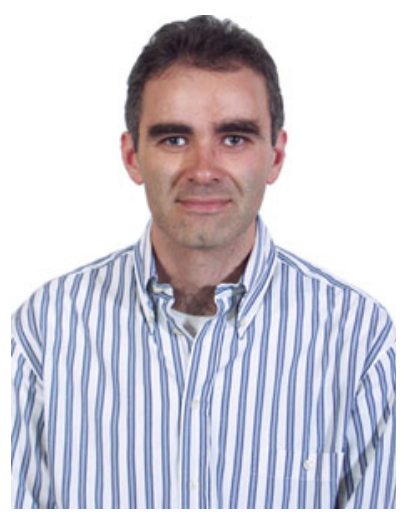

Antonio Cerone received his Laurea degree in information science in 1989 and his $\mathrm{PhD}$ degree in computer science in 1993, both from the University of Pisa, Italy. After holding research positions in Germany and Australia, since 2004 he is Research Fellow at the International Institute for Software Technology (IIST) of the United Nations University (UNU), which is located in Macau SAR, China. His research focuses on education and e-learning as well as on formal methods and their application to interactive systems, system biology, ecosystems, information security, asynchronous hardware, concurrent and real-time systems, and safety-critical systems. Antonio is the leader of the UNU-IIST Programme on "Strategies and Technologies for Education" and coordinates the UNU-IIST Postgraduate Programme. He is the Chair of the Steering Committee of the IEEE International Conference on Software Engineering and Formal Methods (SEFM). He is a member of Formal Methods Europe (FME) and the European Association for Software Science and Technology (EASST). $\mathrm{He}$ is also Editorial Board Member of Electronic Communications of the EASST (ECEASST).

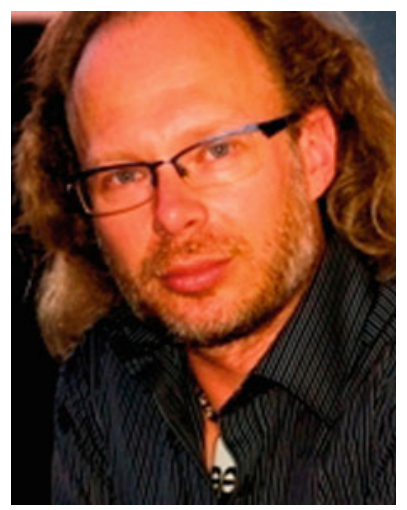

Stefan Gruner (http://www. stefan-gruner.de) received this MSc (Dipl.-Inf.) degree in computer science in 1996 and his $\mathrm{PhD}$ degree (Dr.rer.nat.) in software engineering in 1999, both from the RWTH Aachen, Germany. After some post-doc years in Germany, France and Great Britain, Stefan Gruner is currently a senior lecturer at the Department of Computer Science at the University of Pretoria, South Africa, where he is managing the new SSFM research group on software science and formal methods. Stefan Gruner is a member of the European Association for Software Science and Technology (EASST), the European Association for Programming Languages and Systems (EAPLS), as well as Formal Methods Europe (FME). 